\title{
Does liver cirrhosis affect the surgical outcome of primary colorectal cancer surgery? A meta-analysis
}

\author{
Yu-Xi Cheng ${ }^{\dagger}$, Wei Tao ${ }^{\dagger}$, Hua Zhang, Dong Peng ${ }^{*}$ (D) and Zheng-Qiang Wei
}

\begin{abstract}
Purpose: The purpose of this meta-analysis was to evaluate the effect of liver cirrhosis (LC) on the short-term and long-term surgical outcomes of colorectal cancer (CRC).

Methods: The PubMed, Embase, and Cochrane Library databases were searched from inception to March 23, 2021. The Newcastle-Ottawa Scale (NOS) was used to assess the quality of enrolled studies, and RevMan 5.3 was used for data analysis in this meta-analysis. The registration ID of this current meta-analysis on PROSPERO is CRD42021238042.

Results: In total, five studies with 2485 patients were included in this meta-analysis. For the baseline information, no significant differences in age, sex, tumor location, or tumor T staging were noted. Regarding short-term outcomes, the cirrhotic group had more major complications ( $\mathrm{OR}=5.15,95 \% \mathrm{Cl}=1.62$ to $16.37, \mathrm{p}=0.005)$, a higher reoperation rate $(\mathrm{OR}=2.04,95 \% \mathrm{Cl}=1.07$ to $3.88, \mathrm{p}=0.03)$, and a higher short-term mortality rate $(\mathrm{OR}=2.85,95 \% \mathrm{Cl}=1.93$ to $4.20, p<0.00001)$ than the non-cirrhotic group. However, no significant differences in minor complications (OR= $1.54,95 \% \mathrm{Cl}=0.78$ to $3.02, \mathrm{p}=0.21$ ) or the rate of intensive care unit (ICU) admission ( $\mathrm{OR}=0.76,95 \% \mathrm{Cl}=0.10$ to 5.99 , $\mathrm{p}=0.80$ ) were noted between the two groups. Moreover, the non-cirrhotic group exhibited a longer survival time than the cirrhotic group ( $H R=2.96,95 \% \mathrm{Cl}=2.28$ to $3.85, \mathrm{p}<0.00001)$.
\end{abstract}

Conclusion: Preexisting LC was associated with an increased postoperative major complication rate, a higher rate of re-operation, a higher short-term mortality rate, and poor overall survival following CRC surgery.

Keywords: Liver cirrhosis, Colorectal cancer, Surgical outcome, Meta-analysis

\section{Introduction}

Liver cirrhosis (LC) is a common disease, causing 1.03 million deaths per year worldwide [1]. Alcohol misuse, infection with hepatitis viruses, and nonalcoholic liver disease are the leading causes of LC [2]. The pathological process of LC results from different mechanisms of liver injury, which leads to necroinflammation and fibrogenesis of liver tissues [3]. Moreover, the morbidity

\footnotetext{
* Correspondence: carry dong@126.com

${ }^{\dagger}$ Yu-Xi Cheng and Wei Tao contributed equally to this work. Department of Gastrointestinal Surgery, The First Affiliated Hospital of Chongqing Medical University, Chongqing 400016, China
}

rate and the mortality rate are reported to be increased in patients with LC who undergo non-hepatic abdominal surgeries [4-6].

Colorectal cancer (CRC) is the third most common cancer and the second leading cause of cancer-related deaths worldwide [7]. Radical resection of the colorectal tumors has been widely accepted as a curative treatment [8]. Tumor location, comorbidity, clinical stage, anastomosis methods, and total mesorectal excision are reported to have an effect on the outcome of CRC surgery [9-12].

(c) The Author(s). 2021 Open Access This article is licensed under a Creative Commons Attribution 4.0 International License, which permits use, sharing, adaptation, distribution and reproduction in any medium or format, as long as you give appropriate credit to the original author(s) and the source, provide a link to the Creative Commons licence, and indicate if changes were made. The images or other third party material in this article are included in the article's Creative Commons licence, unless indicated otherwise in a credit line to the material. If material is not included in the article's Creative Commons licence and your intended use is not permitted by statutory regulation or exceeds the permitted use, you will need to obtain permission directly from the copyright holder. To view a copy of this licence, visit http://creativecommons.org/licenses/by/4.0/ The Creative Commons Public Domain Dedication waiver (http://creativecommons.org/publicdomain/zero/1.0/) applies to the data made available in this article, unless otherwise stated in a credit line to the data. 
A recent study reported that LC had strong associations with the colorectal adenoma-carcinoma sequence [13]; however, the prognoses and outcomes of CRC surgery in patients with $\mathrm{LC}$ remain controversial.

Some studies reported that CRC patients with LC had more postoperative complications [14], but others reported the opposite [15]. In addition, few studies have assessed the specific surgical outcomes and perioperative management in patients with CRC. Thus, the purpose of this current meta-analysis was to evaluate the effect of $\mathrm{LC}$ on the short-term and long-term surgical outcomes of CRC.

\section{Methods}

This study design stringently conformed to the Preferred Reporting Items for Systematic Reviews and Meta Analyses (PRISMA) statement [16]. The registration ID of this current meta-analysis on PROSPERO is CRD42021238042, and the link is https://www.crd.york.ac.uk/prospero/display_record. php?ID=CRD42021238042.

\section{Literature search}

We conducted a systematic literature search of PubMed, Embase, and the Cochrane Library, and the last search was performed on March 23, 2021. The following keywords related to cirrhosis were used for the search: (liver cirrhosis) OR (cirrhosis) OR (cirrhotic). The items related to CRC were as follows: (colorectal cancer) OR (colon cancer) OR (rectal cancer) OR (colorectal neoplasm) OR (colon neoplasm) OR (rectal neoplasm) OR (colorectal tumor) OR (colon tumor) OR (rectal tumor). Then, we combined these items with "AND". The search was restricted to publications in English, and two authors performed the search independently.

\section{Inclusion and exclusion criteria}

Eligible studies were identified according to the following inclusion criteria: (1) human subjects and (2) studies reporting a comparison of surgical endpoints between cirrhotic patients and non-cirrhotic patients with CRC. The baseline information in the studies included the following: age, sex, tumor location, and tumor depth. Studies included the following primary or secondary endpoints as follows: major complications, minor complications, postoperative intensive care unit (ICU) admission, and the rate of re-operation or postoperative death. Postoperative complications were classified into two groups (minor complications and major complications) according to the Clavien-Dindo classification [17]. The exclusion criteria were as follows: (1) studies with incomplete data and (2) studies with no relevant endpoints. Case reports, case series, comments, letters to the editor, conference abstracts, and non-original articles were excluded.

\section{Study selection}

All of the selected studies were screened by the same two authors. First, the titles and abstracts were screened to assess eligibility for inclusion in this meta-analysis. Then, the full texts were carefully checked to make final decisions based on the inclusion and exclusion criteria. For potential studies, an extensive manual search of relevant studies from the reference lists was conducted. Disagreements were resolved by a third senior author.

\section{Data extraction}

Two authors extracted the data separately. The following data were collected: first author, year of publication, period of study, region of study, sample size, baseline information, major complications, minor complications, postoperative ICU admission, rate of re-operation, and postoperative death. The unpublished or unclear data were accessed by contacting the original authors through email if applicable. Then, the overall survival data were extracted by using the software Engauge Digitizer [18], with as many coordinate points taken as possible. Discussions were conducted in groups if disagreements occurred.

\section{Surgical outcomes}

The surgical outcomes included the main outcomes and the secondary outcomes. The main outcomes of the current meta-analysis were postoperative complications, including minor complications and major complications. The secondary outcomes were postoperative ICU admission, the rate of re-operation, the short-term mortality rate, and long-term survival.

\section{Quality assessment}

The Newcastle-Ottawa Scale (NOS), which has a score ranging from zero to nine points, was used to assess the quality of the enrolled studies. Three domains, selection, comparability, and results, were evaluated for each study [19]. A study with a score of nine points was considered high quality, a study with a score of seven to eight points was considered medium quality, and a study with six or less was considered low quality. The senior author completed this assessment independently.

\section{Statistical analysis}

The mean differences (MDs) and 95\% confidence intervals (CIs) were calculated for age. The odds ratios (ORs) and $95 \%$ CIs were calculated for sex, tumor location, tumor depth, and surgical outcomes. The statistical heterogeneity for the included studies was evaluated by using the $\mathrm{I}^{2}$ value. The random effects model was used when $I^{2}>50 \%$, which was considered to indicate high heterogeneity, and $\mathrm{p}<0.1$, which was considered to indicate statistical significance. Otherwise, the fixed effects 
model was used, and $\mathrm{p}<0.05$ was considered statistically significant [20]. RevMan 5.3 (The Cochrane Collaboration, London, UK) was used to perform the data analysis in this meta-analysis.

\section{Results}

\section{Study selection}

From an initial total of 1096 studies identified in the databases (389 studies in PubMed, 693 studies in Embase, and 14 studies in the Cochrane Library), 293 were removed due to duplication. After screening the titles and abstracts, 16 studies underwent a full-text review. Finally, five studies $[4,14,15,21,22]$ were included in this meta-analysis. The flow chart of article selection is shown in Fig 1.

\section{Patient characteristics and quality assessment of the included studies}

In total, five studies with 2485 patients were included in this meta-analysis. All studies were retrospective studies from diverse regions, including Romania, France, Denmark, and Korea. The year of publication ranged from 2013 to 2020, and the study date ranged from 1996 to 2014. The grade of complications and the NOS score are shown in Table 1.

\section{Baseline information}

Age, sex, tumor location, and tumor depth were extracted as baseline information. The results showed no significant differences in sex $(\mathrm{OR}=1.28,95 \% \mathrm{CI}=0.92$ to $\left.1.79, \mathrm{I}^{2}=54 \%, p=0.14\right)$, age ( $\mathrm{MD}=0.06,95 \% \mathrm{CI}=-0.93$ to $1.05, \mathrm{I}^{2}=0 \%, p=0.91$ ), tumor location (colon: $\mathrm{OR}=0.82$, 95\% $\mathrm{CI}=0.62$ to $1.09, \mathrm{I}^{2}=0 \%, p=0.17$; rectal: $\mathrm{OR}=1.21$, $95 \% \mathrm{CI}=0.91$ to $\left.1.61, \mathrm{I}^{2}=0 \%, p=0.18\right)$, or tumor $\mathrm{T}$ staging (T1-T2: $\mathrm{OR}=1.14,95 \% \mathrm{CI}=0.77$ to $1.69, \mathrm{I}^{2}=69 \%, p=0.52$; T3-T4: $\mathrm{OR}=0.81,95 \% \mathrm{CI}=0.59$ to $1.12, \mathrm{I}^{2}=53 \%, p=0.20$ ) between the cirrhotic group and non-cirrhotic group (Table 2).

\section{Complications}

Three studies $[4,14,21]$ investigated minor complications, and no significance was observed between the two groups $\left(\mathrm{OR}=1.54,95 \% \mathrm{CI}=0.78\right.$ to $3.02, \mathrm{I}^{2}=54 \%, p=0.21$ ) (Fig. 2a). However, in terms of major complications in the same three studies $[4,14,21]$, the cirrhotic group had more major complications than the non-cirrhotic group (OR=5.15, 95\% $\mathrm{CI}=1.62$ to $16.37, \mathrm{I}^{2}=71 \%, p=$ 0.005) (Fig. 2b).

\section{Postoperative ICU admission}

Two studies [4, 15] including 1634 participants reported postoperative ICU admission. The results indicated no difference between the cirrhotic group and non-cirrhotic

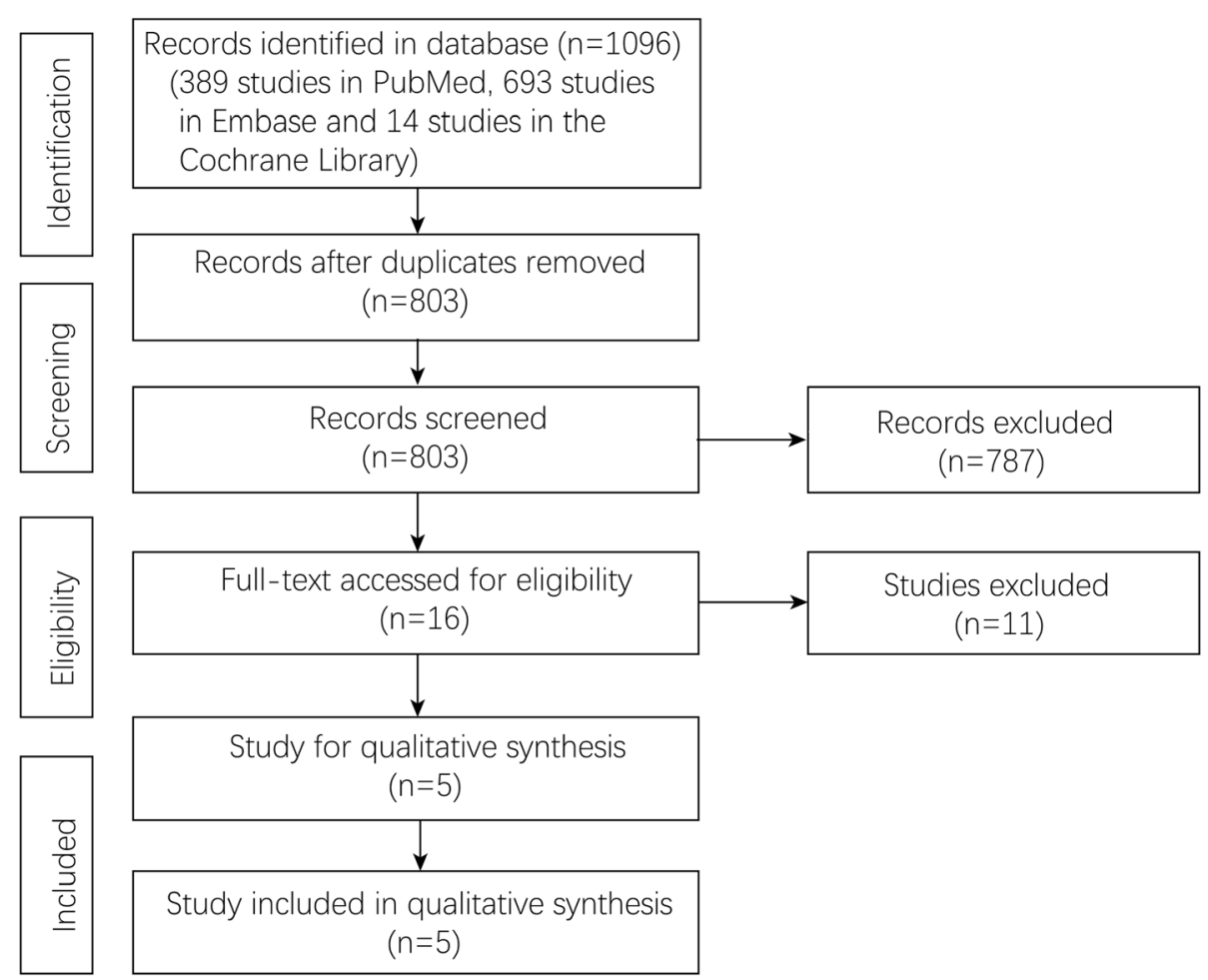

Fig. 1 Flowchart of study selection 
Table 1 Characteristics of the studies included in the meta-analysis

\begin{tabular}{|c|c|c|c|c|c|c|c|c|c|}
\hline \multirow[t]{2}{*}{ Author } & \multirow[t]{2}{*}{$\begin{array}{l}\text { Year } \\
\text { published }\end{array}$} & \multirow[t]{2}{*}{ Country } & \multirow[t]{2}{*}{$\begin{array}{l}\text { Study } \\
\text { design }\end{array}$} & \multirow[t]{2}{*}{$\begin{array}{l}\text { Study } \\
\text { date }\end{array}$} & \multicolumn{2}{|c|}{ Sample size } & \multicolumn{2}{|c|}{$\begin{array}{l}\text { Postoperative complications } \\
\text { Clavien-Dindo classification (I/II/ } \\
\text { III/IV/V) }\end{array}$} & \multirow[t]{2}{*}{ NOS } \\
\hline & & & & & $\begin{array}{l}\text { Cirrhotic } \\
\text { group }\end{array}$ & $\begin{array}{l}\text { Non-cirrhotic } \\
\text { group }\end{array}$ & $\begin{array}{l}\text { Cirrhotic } \\
\text { group }\end{array}$ & $\begin{array}{l}\text { Non-cirrhotic } \\
\text { group }\end{array}$ & \\
\hline Shin et al. [15] & 2020 & Korea & Retrospective & $\begin{array}{l}2008- \\
2013\end{array}$ & 453 & 906 & $\|/\| \|$ & $\|/\| I \|$ & 8 \\
\hline Lacatus et al. [21] & 2018 & Romania & Retrospective & $\begin{array}{l}2005- \\
2014\end{array}$ & 68 & 136 & I/I/III/IVN & |/II/II/IVN & 7 \\
\hline Han et al. [4] & 2017 & Korea & Retrospective & $\begin{array}{l}2002- \\
2010\end{array}$ & 55 & 220 & Unknown & Unknown & 7 \\
\hline $\begin{array}{l}\text { Sabbagh et al. } \\
\text { [14] }\end{array}$ & 2016 & France & Retrospective & $\begin{array}{l}2006- \\
2014\end{array}$ & 40 & 80 & Unknown & Unknown & 7 \\
\hline $\begin{array}{l}\text { Montomoli et al. } \\
\text { [22] }\end{array}$ & 2013 & Denmark & Retrospective & $\begin{array}{l}1996- \\
2009\end{array}$ & 158 & 369 & III/IVN & III/IVN & 8 \\
\hline
\end{tabular}

Abbreviations: NOS Newcastle-Ottawa Scale

group (OR=0.76, $95 \% \mathrm{CI}=0.10$ to $5.99, \mathrm{I}^{2}=94 \%, p=0.80$ ) (Fig. 2c).

\section{Reoperation rate}

Two studies [14, 21] investigated the rate of reoperation, and the cirrhotic group showed a higher rate of re-operation than the non-cirrhotic group $(\mathrm{OR}=2.04$, $95 \% \mathrm{CI}=1.07$ to $3.88, \mathrm{I}^{2}=0 \%, \mathrm{p}=0.03$ ) (Fig. $2 \mathrm{~d}$ ).

\section{Short-term mortality rate}

All five studies [4, 14, 15, 21, 22] including 2485 patients reported the short-term mortality rate. The cirrhotic group had a higher death rate than the non-cirrhotic group (OR=2.85, 95\% $\mathrm{CI}=1.93$ to $4.20, \mathrm{I}^{2}=43 \%, \quad p<$ 0.00001) (Fig. 3).

\section{Long-term survival}

Three articles $[4,14,15]$ reported long-term survival. The non-cirrhotic group had a better overall survival than the cirrhotic group (HR=2.96, 95\% $\mathrm{CI}=2.28$ to 3.85 , $\mathrm{I}^{2}=0 \%, p<0.00001$ ) (Fig. 4).

\section{Sensitivity and publication bias}

Repeated meta-analysis was performed by excluding one study at a time, and the exclusion of any one study did not significantly alter the results. Publication bias for the included studies was based on a visual inspection of the funnel plot. The funnel plot was symmetrical, and no obvious publication bias was found (Fig. 5).

\section{Discussion}

LC is a serious comorbidity in cancer-related diseases. Previous studies found that LC had a negative effect on CRC surgery $[14,15]$. In this meta-analysis, five studies with 2485 patients were included. Regarding baseline information, no significant difference was found in terms of age, sex, tumor location, or tumor $\mathrm{T}$ staging between the two groups. Regarding short-term outcomes, the cirrhotic group had more major complications, a higher rate of re-operation, and a higher short-term mortality rate than the non-cirrhotic group. However, minor complications and the ICU admission rate did not significantly differ between the two groups. Moreover, the non-cirrhotic group showed a longer survival time than the cirrhotic group.

Table 2 Summary meta-analysis of comparison between cirrhotic group and non-cirrhotic group

\begin{tabular}{|c|c|c|c|c|}
\hline Subgroup & Studies & Participants (cirrhotic/non-cirrhotic) & Odds ratio/mean difference ( $95 \% \mathrm{Cls})$ & Heterogeneity \\
\hline \multicolumn{5}{|l|}{ Baseline information } \\
\hline Age, year & 2 & $521 / 1042$ & $0.06(-0.93,1.05) ; p=0.91$ & $\mathrm{I}^{2}=0 \% ; p=0.78$ \\
\hline Male & 5 & $774 / 1711$ & $1.28(0.92,1.79) ; p=0.14$ & $I^{2}=54 \% ; p=0.07$ \\
\hline Tumor location colon & 4 & $321 / 805$ & $0.82(0.62,1.09) ; p=0.17$ & $I^{2}=0 \% ; p=0.61$ \\
\hline Tumor location rectal & 4 & $321 / 805$ & $1.21(0.91,1.61) ; p=0.18$ & $P^{2}=0 \% ; p=0.64$ \\
\hline \multicolumn{5}{|l|}{ Tumor staging } \\
\hline $\mathrm{T} 1-\mathrm{T} 2$ & 4 & $706 / 1575$ & $1.14(0.77,1.69) ; p=0.52$ & $I^{2}=69 \% ; p=0.02$ \\
\hline T3-T4 & 4 & $706 / 1575$ & $0.81(0.59,1.12) ; p=0.20$ & $\mathrm{I}^{2}=53 \% ; p=0.09$ \\
\hline
\end{tabular}




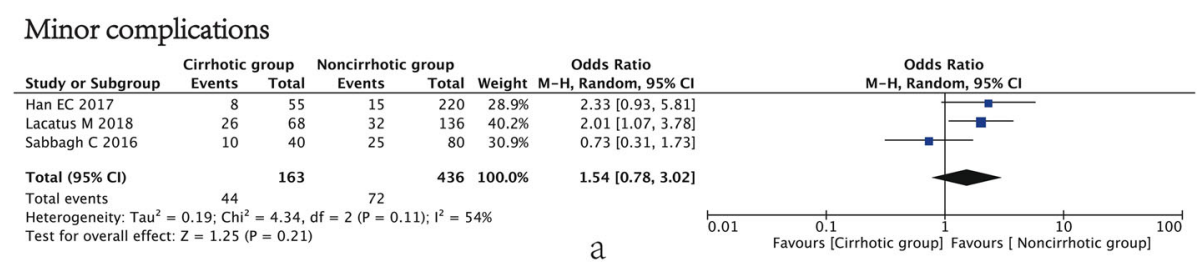

Major complications

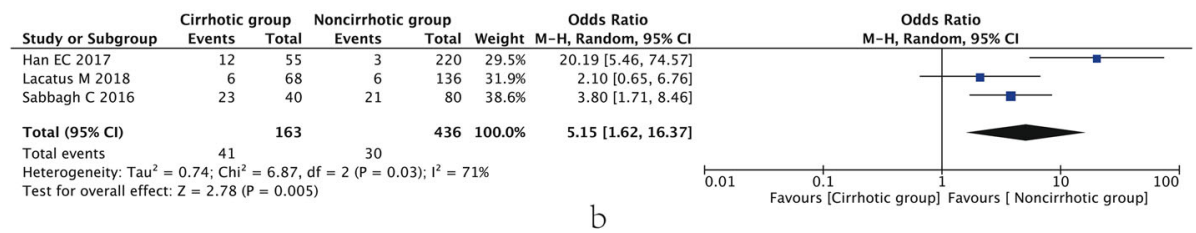

Postoperative ICU admission

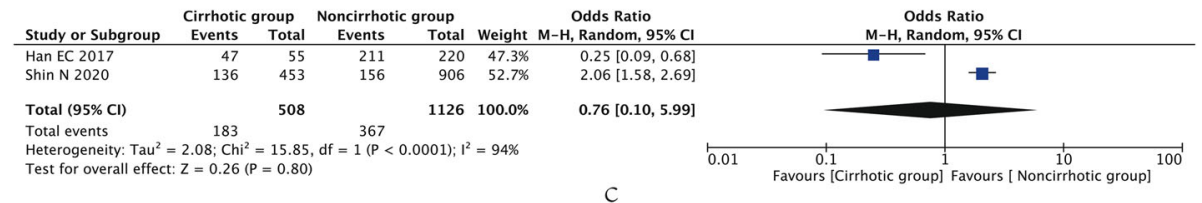

Reoperation rate

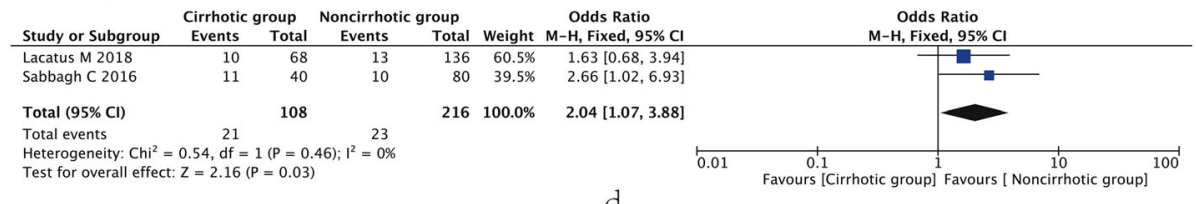

d

Fig. 2 Forest plot showing the short-term outcomes. a Minor complications, b major complications, c postoperative ICU admission, and d Reoperation rate

LC is a chronic disease with many complications, including variceal bleeding, ascites, hepatorenal syndrome, hepatic encephalopathy, and hepatocellular carcinoma in the decompensated stage [23]. Patients with LC suffer from economic burdens and a shortened life expectancy $[24,25]$. A high risk of LC was noted among patients with cirrhosis undergoing abdominal surgery [26]; however, LC was not a contraindication for abdominal surgery or chemotherapy [27]. The preoperative albumin level is correlated with surgical outcomes related to refractory ascites [28]. Previous studies reported that patients with LC undergoing gastrectomy had more complications and a higher mortality rate short term [29, 30]. In this meta- analysis, the cirrhotic group had more major complications than the non-cirrhotic group after CRC surgery. However, no significant difference in minor complications was noted between the two groups. The reasons for this might be the reduced recuperative capacity of the patients, reduced drug metabolism in the liver, and weakened immune function [29-31]. In addition, more major complications could cause a higher rate of re-operation, which is consistent with the results of this meta-analysis. The reason for the higher rate of re-operation in CRC patients with LC might be the inadequate preoperative adjustment of liver function. Hyperbilirubinemia, prothrombin time prolongation, and intraoperative transfusion have been

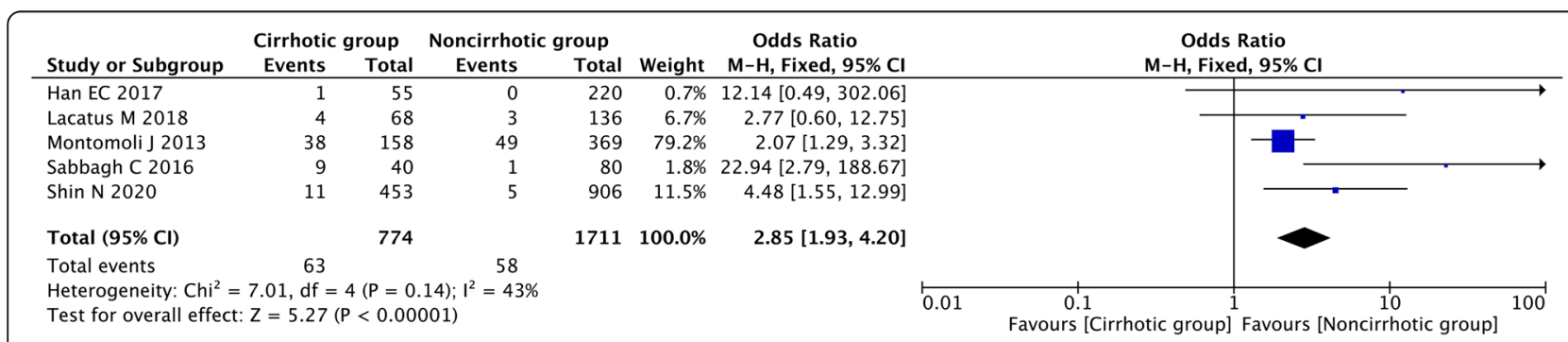

Fig. 3 Forest plot showing the short-term mortality rate 


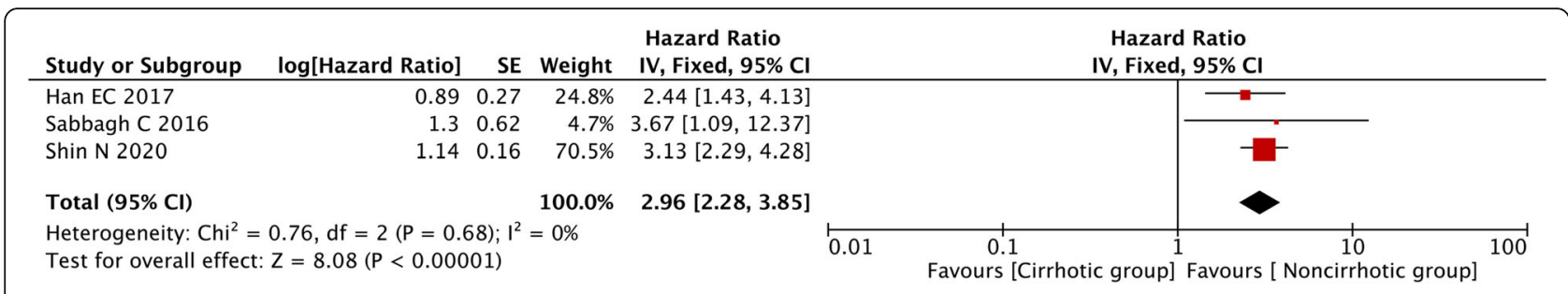

Fig. 4 Forest plot showing the long-term survival

reported as risk factors for postoperative complications in these patients [32]. Therefore, the perioperative management of LC patients who undergo surgery for CRC is crucial.

CRC has already become the second most common cancer-related disease worldwide [7], and patients with concurrent LC and CRC might have a higher death rate after surgery. Previous studies showed that the mortality of the LC group ranged from 18 to $26 \%$, which was higher than that of the non-LC group, and the differences among studies might be due to the patients being treated at community hospitals or specialized hospitals [33, 34]. We observed that the cirrhotic group had a higher short-term mortality rate and a shorter survival time than the noncirrhotic group in this meta-analysis. A possible reason for finding is that decreased liver function could cause hepatic coagulopathy, lower albumin levels, and abnormal liver metabolism, which led to more severe complications, including ascites, infection, bleeding, and anastomotic fistulas [5]. The major complications might be related to short-term death after CRC surgery. Moreover, cirrhosis and intestinal dysfunction cause chronic malnutrition, and LC patients have a high risk for primary hepatic carcinoma [2], which might influence long-term survival.

Before patients with confirmed or suspected LC undergo CRC surgery, a preoperative assessment of liver function is needed to clarify its severity, which should be evaluated by either the Child-Pugh classification (CTP) or Model for End Stage Liver Disease (MELD) score in clinical practice $[35,36]$. A previous study compared CRC patients with different CTPs in terms of surgical outcomes and found that Child B patients had a high rate of complications, more reinterventions, and a longer hospitalization stay [21]. Although classification or risk stratification is vital before surgery in CRC patients with LC, it was lacking in the included studies. The lack of a liver function assessment limited the ability to guide preoperative decisions regarding who underwent CRC surgery, which would require knowing the severity of cirrhosis. Thus, studies on CRC patients with LC should be more comprehensive and include the CTP or MELD score.

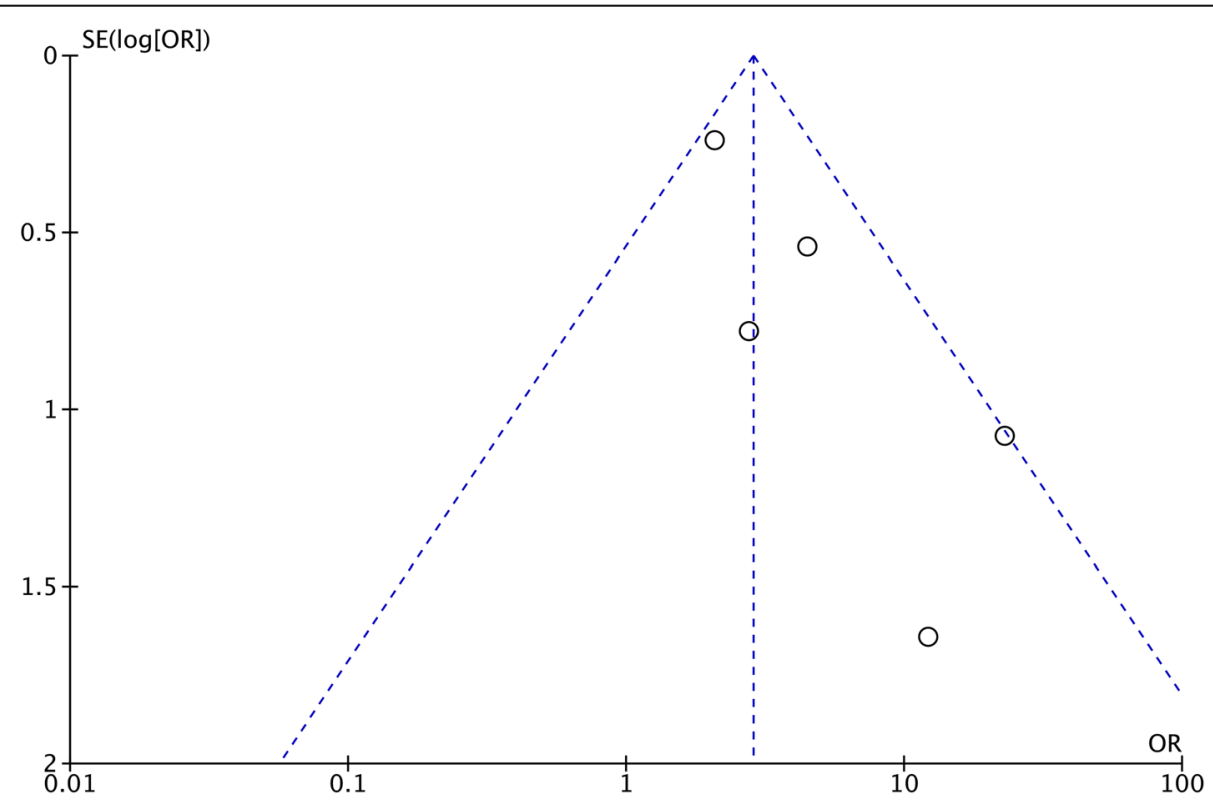

Fig. 5 Funnel plot showing the short-term mortality rate 
There were several strengths of our meta-analysis. First, this meta-analysis was the first to exclusively pool all of the data to evaluate the effect of LC on the shortterm and long-term surgical outcomes of CRC. Second, the controversy about surgical outcomes and long-term survival between the cirrhotic patients and non-cirrhotic patients was settled in this meta-analysis. Third, the present meta-analysis provided some information that will be useful in clinical practice. Perioperative management should be handled cautiously by surgeons among patients with LC, and furthermore, patients should be cautious during the postoperative period due to poor overall survival.

However, certain limitations to this current metaanalysis existed. First, only five studies were included, all of which were retrospective studies; however, no publication bias was shown in the funnel plot. Second, cirrhosis was not classified in each study, and compensated LC and decompensated LC might have different outcomes after CRC surgery. Third, liver function was graded by the CTP or MELD score in only one study, which might have influence on the accuracy of the results. Finally, the long-term survival was analyzed with data from only three studies, which might have increased the clinical heterogeneity. Therefore, comprehensive, prospective, and high-quality randomized controlled trials should be performed in the future.

In conclusion, preexisting LC was associated with an increased postoperative major complication rate, a higher rate of re-operation, a higher short-term mortality rate, and poorer overall survival following CRC surgery. Therefore, surgeons should be careful when conducting CRC surgery on patients with LC.

\section{Abbreviations}

LC: Liver cirrhosis; CRC: Colorectal cancer; ICU: Intensive care unit; NOS: Newcastle-Ottawa Scale; PRISMA: Preferred Reporting Items for Systematic Reviews and Meta Analyses; CTP: Child-Pugh classification; MELD: Model for End Stage Liver Disease

\section{Acknowledgements}

We acknowledge all of the authors whose publications are referred to in our article, and we acknowledge Peng Hong, who helped to edit the English text and revise typographical errors.

\section{Authors' contributions}

Data extraction, YXC and WT; quality assessments, DP; data analysis, DP and $Y X C$; writing —original draft, YXC; writing — review and editing, DP, YXC, WT, $\mathrm{HZ}$, and ZQW. The authors read and approved the final manuscript.

\section{Funding}

This study was supported by Chongqing key diseases Research and Application Demonstration Program (Colorectal Cancer Prevention and Treatment Technology Research and Application Demonstration [No. 2019ZX003]).

\section{Availability of data and materials}

All data generated or analyzed during this study are included in this published article.

\section{Declarations}

Ethics approval and consent to participate

Not applicable.

\section{Consent for publication}

Not applicable.

\section{Competing interests}

The authors declare that they have no competing interests.

Received: 15 February 2021 Accepted: 18 May 2021

Published online: 09 June 2021

References

1. Lozano R, Naghavi M, Foreman K, Lim S, Shibuya K, Aboyans V, et al. Global and regional mortality from 235 causes of death for 20 age groups in 1990 and 2010: a systematic analysis for the Global Burden of Disease Study 2010. Lancet. 2012;380(9859):2095-128. https://doi.org/10.1016/S0140-673 6(12)61728-0.

2. Tsochatzis EA, Bosch J, Burroughs AK. Liver cirrhosis. Lancet. 2014;383(9930): 1749-61. https://doi.org/10.1016/S0140-6736(14)60121-5.

3. Garcia-Tsao G, Friedman S, Iredale J, Pinzani M. Now there are many (stages) where before there was one: in search of a pathophysiological classification of cirrhosis. Hepatology. 2010;51(4):1445-9. https://doi.org/1 0.1002/hep.23478

4. Han EC, Ryoo SB, Park JW, Yi JW, Oh HK, Choe EK, et al. Oncologic and surgical outcomes in colorectal cancer patients with liver cirrhosis: a propensity-matched study. PLoS One. 2017;12(6):e0178920. https://doi.org/1 0.1371/journal.pone.0178920.

5. Lopez-Delgado JC, Ballus J, Esteve F, Betancur-Zambrano NL, Corral-Velez V, Mañez R, et al. Outcomes of abdominal surgery in patients with liver cirrhosis. World J Gastroenterol. 2016;22(9):2657-67. https://doi.org/10.3748/ wjg.v22.i9.2657.

6. del Olmo JA, Flor-Lorente B, Flor-Civera B, Rodriguez F, Serra MA, Escudero $A$, et al. Risk factors for nonhepatic surgery in patients with cirrhosis. World J Surg. 2003;27(6):647-52. https://doi.org/10.1007/s00268-003-6794-1.

7. Bray F, Ferlay J, Soerjomataram I, Siegel RL, Torre LA, Jemal A. Global cancer statistics 2018: GLOBOCAN estimates of incidence and mortality worldwide for 36 cancers in 185 countries. CA Cancer J Clin. 2018;68(6):394-424. https://doi.org/10.3322/caac.21492.

8. Kekelidze M, D'Errico L, Pansini M, Tyndall A, Hohmann J. Colorectal cancer: current imaging methods and future perspectives for the diagnosis, staging and therapeutic response evaluation. World J Gastroenterol. 2013;19(46): 8502-14. https://doi.org/10.3748/wjg.v19.i46.8502.

9. Planellas P, Farrés R, Cornejo L, Rodríguez-Hermosa Jl, Pigem A, Timoteo A, et al. Randomized clinical trial comparing side to end vs end to end techniques for colorectal anastomosis. Int J Surg. 2020;83:220-9. https://doi. org/10.1016/j.jijsu.2020.09.039.

10. Teresa G, Ugo P, Francesca G, et al. Colonic J-Pouch or straight colorectal reconstruction after low anterior resection for rectal cancer: impact on quality of life and bowel function: a multicenter prospective randomized study. Dis Colon Rectum. 2020;63(11):1511-23. https://doi.org/10.1097/DCR. 0000000000001745

11. Liao CK, Chern YJ, Lin YC, Hsu YJ, Chiang JM, Tsai WS, et al. Short- and medium-term outcomes of intracorporeal versus extracorporeal anastomosis in laparoscopic right colectomy: a propensity score-matched study. World J Surg Oncol. 2021;19(1):6. https://doi.org/10.1186/s12957-02002112-2.

12. Lai IL, You JF, Chern YJ, Tsai WS, Chiang JM, Hsieh PS, et al. Survival analysis of local excision vs total mesorectal excision for middle and low rectal cancer in pT1/pT2 stage and intermediate pathological risk. World J Surg Oncol. 2019;17(1):212. https://doi.org/10.1186/s12957-019-1763-9.

13. Troschel AS, Miks A, Troschel FM, Hüsing-Kabar A, Maschmeier M, Heinzow $\mathrm{HS}$, et al. Chronic liver disease promotes lesions of the colorectal adenoma-carcinoma sequence, independent of liver cirrhosis. United European Gastroenterol J. 2019;7(5):662-72. https://doi.org/10.1177/2 050640619826391.

14. Sabbagh C, Chatelain D, Nguyen-Khac E, Rebibo L, Joly JP, Regimbeau JM. Management of colorectal cancer in patients with cirrhosis: a retrospective, 
case-matched study of short- and long-term outcomes. Dig Liver Dis. 2016; 48(4):429-34. https://doi.org/10.1016/j.dld.2015.12.004.

15. Shin N, Han EC, Won S, Ryoo SB, Choe EK, Park BK, et al. The prognoses and postoperative outcomes of patients with both colorectal cancer and liver cirrhosis based on a nationwide cohort in Korea. Ann Surg Treat Res. 2020; 99(2):82-9. https://doi.org/10.4174/astr.2020.99.2.82.

16. Shamseer L, Moher D, Clarke M, Ghersi D, Liberati A, Petticrew M, et al. Preferred reporting items for systematic review and meta-analysis protocols (PRISMA-P) 2015: elaboration and explanation. BMJ. 2015;350:g7647. https:// doi.org/10.1136/bmj.g7647.

17. Dindo D, Demartines N, Clavien PA. Classification of surgical complications: a new proposal with evaluation in a cohort of 6336 patients and results of a survey. Ann Surg. 2004;240(2):205-13. https://doi.org/10.1097/01.sla.0000133 083.54934.ae.

18. Tierney JF, Stewart LA, Ghersi D, Burdett S, Sydes MR. Practical methods for incorporating summary time-to-event data into meta-analysis. Trials. 2007; 8(1):16. https://doi.org/10.1186/1745-6215-8-16

19. Stang A. Critical evaluation of the Newcastle-Ottawa scale for the assessment of the quality of nonrandomized studies in meta-analyses. Eur J Epidemiol. 2010;25(9):603-5. https://doi.org/10.1007/s10654-010-9491-z.

20. Ioannidis JP. Interpretation of tests of heterogeneity and bias in metaanalysis. J Eval Clin Pract. 2008;14(5):951-7. https://doi.org/10.1111/j.1365-2 753.2008.00986.x.

21. Lacatus $M$, Costin $L$, Bodean $V$, et al. The outcome of colorectal surgery in cirrhotic patients: a case match report. Chirurgia (Bucur). 2018; 113(2): 210217. doi: https://doi.org/10.21614/chirurgia.113.2.210.

22. Montomoli J, Erichsen R, Christiansen CF, Ulrichsen SP, Pedersen L, Nilsson T, et al. Liver disease and 30-day mortality after colorectal cancer surgery: a Danish population-based cohort study. BMC Gastroenterol. 2013;13(1):66. https://doi.org/10.1186/1471-230X-13-66.

23. Sturm L, Rössle M, Schultheiß M. Complications of liver cirrhosis pharmaceutical versus interventional therapy. Dtsch Med Wochenschr. 2019. 144(18):1259-66. https://doi.org/10.1055/a-0753-6051.

24. Chung W, Jo C, Chung WJ, Kim DJ. Liver cirrhosis and cancer: comparison of mortality. Hepatol Int. 2018;12(3):269-76. https://doi.org/10.1007/s12072018-9850-5.

25. Blachier M, Leleu H, Peck-Radosavljevic M, Valla DC, Roudot-Thoraval F. The burden of liver disease in Europe: a review of available epidemiological data. J Hepatol. 2013;58(3):593-608. https://doi.org/10.1016/j.jhep.2012.12. 005.

26. Garrison RN, Cryer HM, Howard DA, et al. Clarification of risk factors for abdominal operations in patients with hepatic cirrhosis. Ann Surg. 1984 199(6):648-55. https://doi.org/10.1097/00000658-198406000-00003.

27. Weiss A, Ward EP, Baumgartner JM, Lowy AM, Kelly KJ. Cirrhosis is not a contraindication to cytoreductive surgery and hyperthermic intraperitoneal chemotherapy in highly selected patients. World J Surg Oncol. 2018;16(1): 87. https://doi.org/10.1186/s12957-018-1389-3.

28. Telem DA, Schiano T, Goldstone R, et al. Factors that predict outcome of abdominal operations in patients with advanced cirrhosis. Clin Gastroenterol Hepatol. 2010;8(5):451-7, quiz e58. https://doi.org/10.1016/j. cgh.2009.12.015

29. Wiklund RA. Preoperative preparation of patients with advanced liver disease. Crit Care Med. 2004;32(4 Suppl):S106-15. https://doi.org/10.1097/01. ccm.0000115624.13479.e6.

30. Malik SM, Ahmad J. Preoperative risk assessment for patients with liver disease. Med Clin North Am. 2009;93(4):917-29. https://doi.org/10.1016/j. mcna.2009.03.001

31. Patel T. Surgery in the patient with liver disease. Mayo Clin Proc. 1999;74(6): 593-9. https://doi.org/10.4065/74.6.593.

32. Lee JH, Yu CS, Lee JL, Kim CW, Yoon YS, Park IJ, et al. Factors affecting the postoperative morbidity and survival of patients with liver cirrhosis following colorectal cancer surgery. Int J Colorectal Dis. 2017;32(4):521-30. https://doi.org/10.1007/s00384-016-2739-7.

33. Meunier K, Mucci S, Quentin V, Azoulay R, Arnaud JP, Hamy A. Colorectal surgery in cirrhotic patients: assessment of operative morbidity and mortality. Dis Colon Rectum. 2008;51(8):1225-31. https://doi.org/10.1007/s 1 0350-008-9336-y.

34. Metcalf AM, Dozois RR, Wolff BG, et al. The surgical risk of colectomy in patients with cirrhosis. Dis Colon Rectum. 1987;30(7):529-31. https://doi. org/10.1007/BF02554783
35. O'Leary JG, Friedman LS. Predicting surgical risk in patients with cirrhosis: from art to science. Gastroenterology. 2007;132(4):1609-11. https://doi.org/1 0.1053/j.gastro.2007.03.016

36. Befeler AS, Palmer DE, Hoffman M, Longo W, Solomon H, di Bisceglie AM. The safety of intra-abdominal surgery in patients with cirrhosis: model for end-stage liver disease score is superior to Child-Turcotte-Pugh classification in predicting outcome. Arch Surg. 2005;140(7):650-4; discussion 655. https:// doi.org/10.1001/archsurg.140.7.650

\section{Publisher's Note}

Springer Nature remains neutral with regard to jurisdictional claims in published maps and institutional affiliations.
Ready to submit your research? Choose BMC and benefit from:

- fast, convenient online submission

- thorough peer review by experienced researchers in your field

- rapid publication on acceptance

- support for research data, including large and complex data types

- gold Open Access which fosters wider collaboration and increased citations

- maximum visibility for your research: over $100 \mathrm{M}$ website views per year

At BMC, research is always in progress.

Learn more biomedcentral.com/submissions 